\title{
Primary Assumptions and Guidance Laws in Wave Mechanics
}

\author{
Adriano Orefice*, Raffaele Giovanelli, Domenico Ditto \\ Department of Agricultural and Environmental Sciences (DiSAA), University of Milan, Milan, Italy \\ Email: *adriano.orefice@unimi.it
}

How to cite this paper: Orefice, A., Giovanelli, R. and Ditto, D. (2018) Primary Assumptions and Guidance Laws in Wave Mechanics. Journal of Applied Mathematics and Physics, 6, 2621-2634. https://doi.org/10.4236/jamp.2018.612218

Received: November 20, 2018

Accepted: December 24, 2018

Published: December 27, 2018

Copyright $\odot 2018$ by authors and Scientific Research Publishing Inc. This work is licensed under the Creative Commons Attribution International License (CC BY 4.0).

http://creativecommons.org/licenses/by/4.0/ (c) (i) Open Access

\begin{abstract}
In an article written by Louis de Broglie in 1959 (30 years after the Nobel prize rewarding his foundation of Wave Mechanics), the most challenging problem raised by the Bohr, Heisenberg and Born Standard Quantum Mechanics (SQM) was pointed out in the renunciation to describe "a permanent localization in space, and therefore a well-defined trajectory" for any moving particle. This challenge is taken up in the present paper, showing that de Broglie's Primary Assumption $\boldsymbol{p}=\hbar \boldsymbol{k}$, predicting the wave-particle duality, does also allow to obtain from the energy-dependent form of the Schrödinger and/or Klein-Gordon equations the Guidance Laws piloting particles along well-defined trajectories. The energy-independent equations, on the other hand, may only give rise-both in SQM and in the Bohmian approach-to probabilistic descriptions, overshadowing the role of de Broglie's matter waves in physical space.
\end{abstract}

\section{Keywords}

Helmholtz Equation, Wave Potential, Hamilton-Jacobi Equations, Wave Mechanics, de Broglie's Duality, Matter Waves, Guidance Laws, Schrödinger Equations, Klein-Gordon Equations

\section{Introduction}

We translate here the beginning of a little known de Broglie's paper. "L'interprétation de la Mécanique Ondulatoire" [1], marking his abandonment of the acceptance, lasted 30 years, of the interpretation of Born, Bohr and Heisenberg of Quantum Mechanics and the return to his own original interpretation. The french text is reported in Appendix I.

In my first works on Wave Mechanics, dating back to 1923, I had clearly perceived that it was necessary, in a general way, to associate with the movement 
of any corpuscle the propagation of a wave. But the homogeneous wave that I had been led to consider, and that became the wave $\psi$ of the usual wave mechanics, did not seem to me to describe the physical reality (...). Giving no particular prerogative to any point in space, it was not capable of representing the position of the corpuscle: we could suppose at most, as was very shortly done, that it gave, by its square, the "probability of presence" of the corpuscle in each point (...)

No physicist ignores today (1959) that Wave Mechanics has received for more than thirty years a "purely probabilistic" interpretation in which the wave associated with the corpuscle is no more than a probability representation dependent on the state of our information, and likely to vary abruptly with it (Heisenberg's "reduction of the probability packet"), while the corpuscle is conceived as having no permanent location in space and, consequently, as not describing a well-defined trajectory. This way of conceiving the wave-particle dualism has received the name of "complementarity", a rather vague notion that was tentatively extrapolated, in a somewhat perilous way, outside the realm of physics.

This interpretation of Wave Mechanics, quite different, I will recall, from the one I had considered at the beginning of my research, is mainly due to Born, Bohr and Heisenberg, whose brilliant works are worthy, no doubt, of the greatest admiration. It has been adopted fairly quickly by almost all theorists, despite the express reserves made by such eminent minds as Einstein and Schrödinger and despite their objections. Personally, after having proposed a radically different interpretation, I joined the one that had become "orthodox", and I taught it for many years. Since 1951, however, in particular after the attempts made at that time by Bohm and Vigier, I asked myself, once more, if my original orientation towards the problem posed by the existence of the wave-corpuscule dualism could be the good one.

A few years have passed, and it seems to me that the time has come for a new review of the state of the question, taking into account the progress made since my 1953-54 presentations.

The strongest objections that can be raised against the currently accepted interpretation of wave mechanics concern the non-localization of the corpuscle in this interpretation. It admits, indeed, that, if the state of our knowledge on a corpuscle is represented by an extended wave-train, the corpuscle is present in all the points of this wave-train with a probability [density] equal to $|\psi|^{2}$ : this presence could be qualified as "potential", and it is only at the moment when we notice the presence of the corpuscle at a point of the wave-train by an observation that this potentiality is "actualized"-in the language of philosophers. Such a conception encounters difficulties which have been pointed out with force, and in various ways, by Einstein and Schrödinger; L. de Broglie [1].

As exemplified by [2], Standard Quantum Mechanics (SQM) was generally presented, since the very beginning, by "conveniently assuming as fundamental postulates" the time-dependent Schrödinger equation and its probabilistic inter- 
pretation, "since every department of deductive science must necessarily be founded on certain Primary Assumptions".

We show in the present paper that an answer to de Broglie's problem concerning the particle localization may be obtained from quite simpler, and more evident, Primary Assumptions, suggested by the very foundations of Wave Mechanics.

Section 2 presents the demonstration that any Helmholtz-like equation is associated with a set of exact Hamiltonian "ray trajectories". Section 3 shows that both the Schrödinger and the Klein-Gordon energy-dependent equations, because of their belonging to the Helmholtz-like family, are associated with matter-wave trajectories along which, thanks to de Broglie's Primary Assumption $\boldsymbol{p}=\hbar \boldsymbol{k}$, the particle motion is addressed and piloted. These trajectories provide therefore, both in the non-relativistic and in the relativistic case, the Guidance Laws allowing to solve de Broglie's problem. The energy-dependence of those equations allows an exact dynamic representation of the particle motion, running as close as possible to the corresponding classical description, and basically distinct-as discussed in Sections 4 and 5-from the probabilistic flow lines and Guidance Laws of the Bohmian theory, whose hydrodynamic approach is based on the same logic and Primary Assumptions as SQM.

\section{Helmholtz Ray-Tracing}

Referring to a stationary medium with refractive index $n(\boldsymbol{r}, \omega)$, sustaining an electromagnetic wave of the form

$$
\psi(\boldsymbol{r}, \omega, t)=u(\boldsymbol{r}, \omega) \mathrm{e}^{-i \omega t},
$$

where $u(\boldsymbol{r}, \omega)$ is assumed to be a solution of the Helmholtz equation

$$
\nabla^{2} u+\left(n k_{0}\right)^{2} u=0,
$$

the standard replacement

$$
u(\boldsymbol{r}, \omega)=R(\boldsymbol{r}, \omega) \mathrm{e}^{i \varphi(\boldsymbol{r}, \omega)},
$$

with real amplitude $R(\boldsymbol{r}, \omega)$ and phase $\varphi(\boldsymbol{r}, \omega)$, is easily seen to split Equation (2), after the separation of real and imaginary parts and after the definition of the wave-vector

$$
\boldsymbol{k}=\nabla \varphi(\boldsymbol{r}, \omega)
$$

and of the function

$$
W(\boldsymbol{r}, \omega)=-\frac{c}{2 k_{0}} \frac{\nabla^{2} R(\boldsymbol{r}, \omega)}{R(\boldsymbol{r}, \omega)},
$$

into the equation system

$$
\left\{\begin{array}{l}
\nabla \cdot\left(R^{2} \nabla \varphi\right)=0 \\
D(\boldsymbol{r}, \boldsymbol{k}, \omega) \equiv \frac{c}{2 k_{0}}\left[k^{2}-\left(n k_{0}\right)^{2}\right]+W(\boldsymbol{r}, \omega)=0
\end{array}\right.
$$


The differentiation $\frac{\partial D}{\partial \boldsymbol{r}} \cdot \mathrm{d} \boldsymbol{r}+\frac{\partial D}{\partial \boldsymbol{k}} \cdot \mathrm{d} \boldsymbol{k}=0$ of Equation (7) associates to Equation (2) an exact Hamiltonian ray-tracing system of the form

$$
\left\{\begin{array}{l}
\frac{\mathrm{d} \boldsymbol{r}}{\mathrm{d} t}=\frac{\partial D}{\partial \boldsymbol{k}} \equiv \frac{c \boldsymbol{k}}{k_{0}} \\
\frac{\mathrm{d} \boldsymbol{k}}{\mathrm{d} t}=-\frac{\partial D}{\partial \boldsymbol{r}} \equiv \nabla\left[\frac{c k_{0}}{2} n^{2}(\boldsymbol{r}, \omega)-W(\boldsymbol{r}, \omega)\right] \\
\nabla \cdot\left(R^{2} \boldsymbol{k}\right)=0 \\
|\boldsymbol{k}(t=0)|=k_{0}=\omega / c
\end{array}\right.
$$

whose time-integration provides a stationary ray-trajectory system coupled by the term $W(\boldsymbol{r}, \omega)$ (which we called Wave Potential) acting perpendicularly, at each point, to the relevant ray trajectories, together with the time-table of the "rays" along these trajectories.

The ray-trajectory coupling due to the (monochromatic) Wave Potential is the one and only cause of any diffraction and interference process. When, however, the space variation length $L$ of the wave amplitude $R(\boldsymbol{r}, \omega)$ turns out to satisfy the condition $k_{0} L \gg 1$, Equation (7) reduces to the eikonal equation

$$
k^{2} \equiv(\nabla \varphi)^{2} \simeq\left(n k_{0}\right)^{2},
$$

describing the geometrical optics limit, where the rays are seen to propagate independently from one another, without any diffraction and/or interference process.

In conclusion, any Helmholtz-like equation of the form (2) is associated to a stationary system of exact ray-trajectories, a basic information which was not available until 2009 [3]-[8].

\section{Back to de Broglie's Wave Mechanics}

Let us refer now, indifferently, to non-relativistic or relativistic Dynamics, and let us consider the simple case of non-interacting point-particles of mass $m$, rest mass $m_{0}$ and total energy $E$, launched with an initial momentum $\boldsymbol{p}_{0}$ into an external force field deriving from a time-independent potential energy $V(\boldsymbol{r})$.

The classical non-relativistic dynamics of each particle is summarized, as is well known [9], by the time-independent Hamilton-Jacobi (H-J) equation

$$
(\nabla S)^{2}=2 m[E-V(\boldsymbol{r})],
$$

while the classical relativistic dynamics is summarized by the time-independent Hamilton-Jacobi equation

$$
(\nabla S)^{2}=\left[\frac{E-V(\boldsymbol{r})}{c}\right]^{2}-\left(m_{0} c\right)^{2} .
$$

Both in Equation (10) and Equation (11) the basic property of the H-J function $S(\boldsymbol{r}, E)$ is the fact that the particle momentum is given by

$$
\boldsymbol{p}=\nabla S(\boldsymbol{r}, E) .
$$


Recalling the Fermat and Maupertuis variational principles, Louis de Broglie [10] [11] [12] was induced to associate each particle of momentum $\boldsymbol{p}$ with a suitable "matter wave" (with wave-vector $\boldsymbol{k}$ ) of the form

$$
\psi(\boldsymbol{r}, \omega, t)=u(\boldsymbol{r}, \omega) \mathrm{e}^{-i \omega t} \equiv R(\boldsymbol{r}, \omega) \mathrm{e}^{i[\varphi(\boldsymbol{r}, \omega)-\omega t]}
$$

under Planck's condition

$$
E=\hbar \omega,
$$

according to the Primary Assumption (laying the foundations of Wave Mechanics)

$$
\boldsymbol{p}=\hbar \boldsymbol{k} .
$$

We have therefore, from Equation (4) and Equation (12), the relations

$$
\varphi=S(\boldsymbol{r}, E) / \hbar \text { and } u(\boldsymbol{r}, E) \equiv R(\boldsymbol{r}, E) \mathrm{e}^{\frac{i}{\hbar} S(\boldsymbol{r}, E)},
$$

The scalar form $p=\hbar k$ (whence $\lambda=2 \pi \hbar / p$ ) of de Broglie's Primary Assumption was very soon verified by the Davisson-Germer electron diffraction experiments [13], which established once and for all the physical reality of matter waves and of the wave-particle duality, and was sufficient by itself to grant a Nobel Prize to all of them.

The vector form of Equation (15) appeared, in its turn, together with Equation (16), to be quite eloquent: they told that the H-J surfaces $S(\boldsymbol{r}, E)=$ const represent the phase-fronts of the newly contrived matter waves, and that the particle momentum $\boldsymbol{p}$ is addressed along the wave-vector $\boldsymbol{k}$, orthogonal to the phase-fronts of the relevant matter waves.

The discovery of a satisfactory Guidance Law of the particles along their dynamic trajectories, however, had not yet been reached. An important step in this direction was performed by Schrödinger [14] [15] [16] [17], assuming that the laws of Classical Mechanics (represented here by Equation (10) and Equation (11)) are the eikonal approximation of suitable Helmholtz-like equations of the form (2). By performing therefore, in the non-relativistic case (10), the replacement

$$
\left(n k_{0}\right)^{2} \rightarrow k^{2} \equiv \frac{p^{2}}{\hbar^{2}}=\frac{2 m}{\hbar^{2}}(E-V)
$$

suggested by the eikonal Equation (9), into Equation (2), we get the Helmholtz-like equation

$$
\nabla^{2} u(\boldsymbol{r}, E)+\frac{2 m}{\hbar^{2}}[E-V(\boldsymbol{r})] u(\boldsymbol{r}, E)=0,
$$

which is the so-called "time-independent" (but energy-dependent) Schrödinger equation [18] [19]: an eigen-value equation admitting in general both continuous and discrete eigen-function spectra and energy eigen-values, which bypass the heuristic prescriptions of the "old" quantum theory.

By performing, similarly, in the relativistic case (11), the replacement

$$
\left(n k_{0}\right)^{2} \rightarrow k^{2} \equiv \frac{p^{2}}{\hbar^{2}}=\left[\frac{E-V(\boldsymbol{r})}{\hbar c}\right]^{2}-\left(\frac{m_{0} c}{\hbar}\right)^{2}
$$


into Equation (2), we get the Helmholtz-like equation

$$
\nabla^{2} u(\boldsymbol{r}, E)+\left[\left(\frac{E-V(\boldsymbol{r})}{\hbar c}\right)^{2}-\left(\frac{m_{0} c}{\hbar}\right)^{2}\right] u(\boldsymbol{r}, E)=0,
$$

which is the so-called "time-independent" (but energy-dependent) Klein-Gordon equation (holding even in the case of particles with $m_{0}=0$ ).

In order to perform the final step toward a reliable Guidance Law in the $a b$ sence of further information, de Broglie considered the idea [20] [21] [22] of a non-linear "double-solution" underlying the Schrödinger and Klein-Gordon equations: a theory which did never get, for him, a satisfactory level.

As we know from Section 2, however, we are nowadays informed of the property of Helmholtz-like equations of being associated with exact kinematic sets of ray-trajectories. The reply to de Broglie's question: “can a particle have a permanent localization in space?" appears therefore to be almost immediate. Both in the non-relativistic and in the relativistic case, in fact, we have only to repeat the procedure of Section 2, by replacing the function $u(\boldsymbol{r}, E)$, given by Equation (16), into the Helmholtz-like Equation (18) and/or Equation (20), and by separating, once more, real from imaginary parts.

The long-desired Guidance Law is finally reached in the general form

$$
\frac{\mathrm{d} \boldsymbol{r}}{\mathrm{d} t}=\frac{\partial H}{\partial \boldsymbol{p}}
$$

where the energy function $H(\boldsymbol{r}, \boldsymbol{p}, E)$ takes on the form

$$
H(\boldsymbol{r}, \boldsymbol{p}, E) \equiv \frac{p^{2}}{2 m}+W(\boldsymbol{r}, E)+V(\boldsymbol{r})
$$

in the non-relativistic case, and the form

$$
H(\boldsymbol{r}, \boldsymbol{p}, E) \equiv V(\boldsymbol{r})+\sqrt{(p c)^{2}+\left(m_{0} c^{2}\right)^{2}+2 E W(\boldsymbol{r}, E)}
$$

in the relativistic case. In both cases, the particle trajectories and time-tables are found by time-integrating Equation (21) in parallel with the relevant Hamiltonian system of dynamical equations reported in Appendix II. Not by sheer coincidence Equation (21) takes on, in the relativistic case, the same form as de Broglie's Guidance Law of [20] [21] [22], showing that, in spite of his dissatisfaction, he wasn't far from his goal. The missing pieces of the puzzle, in his approach, were the equations accompanying Equation (21) in the Hamiltonian systems AII-1 and/or AII-2, and determining the relevant Helmholtz trajectories. In both systems, in particular, a suitable Wave Potential function $W(\boldsymbol{r}, E)$, acting orthogonally to the particle motion and exerting therefore an energy-conserving "gentle drive", is seen to be the cause of any diffraction and/or interference wave-mechanical process.

The time-integration of the wave-mechanical systems AII-1 andlor AII-2 provides, in conclusion, de Broglie's missing link, without any further assumption and without resorting to any kind of probabilistic interpretation. The particle trajectories and time-tables are simply found, in fact [8], by assigning $\boldsymbol{r}(E, t=0)$, 
$\boldsymbol{p}(E, t=0)$ and $R(\boldsymbol{r}, E, t=0)$ over a suitable launching surface, and making use of the equation (see both AII-1 and AII-2)

$$
\nabla \cdot\left(R^{2} \boldsymbol{p}\right)=0,
$$

expressing the constancy of the flux of $R^{2} \boldsymbol{p}$ along any tube formed by the trajectories, in order to obtain the wave amplitude $R(\boldsymbol{r}, E)$ and the Wave Potential function $W(\boldsymbol{r}, E)$ at each time-step. The energy-dependence of Equation (18) and Equation (20) provides, moreover, a crucial analogy with Classical Mechanics, allowing to build up exact trajectories unfolding as close as possible to the relevant classical limits, to which they reduce when the Wave Potential term is neglected, i.e. in their eikonal approximation.

Let us consider for instance, in Figure 1 and Figure 2, the non-relativistic case of a particle beam of the initial Gaussian form $R(x ; z=0) \propto \exp \left(-x^{2} / w_{0}^{2}\right)$, with half-width $w_{0}$, launched along the z-axis, from the left hand side, into a potential field $V(x, z)$ representing a lens-like focalizing structure [8]. The point-like focus (Figure 1) obtained in the eikonal limit, i.e. according to Classical

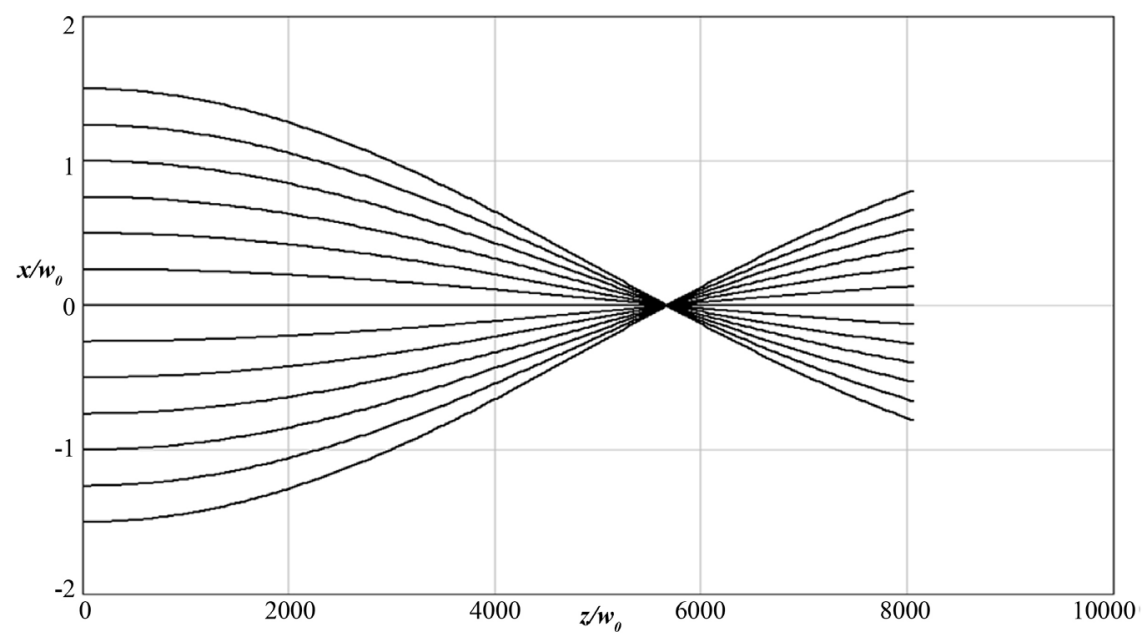

Figure 1. Lens-like potential: point-like focusing in the absence of Wave Potential.

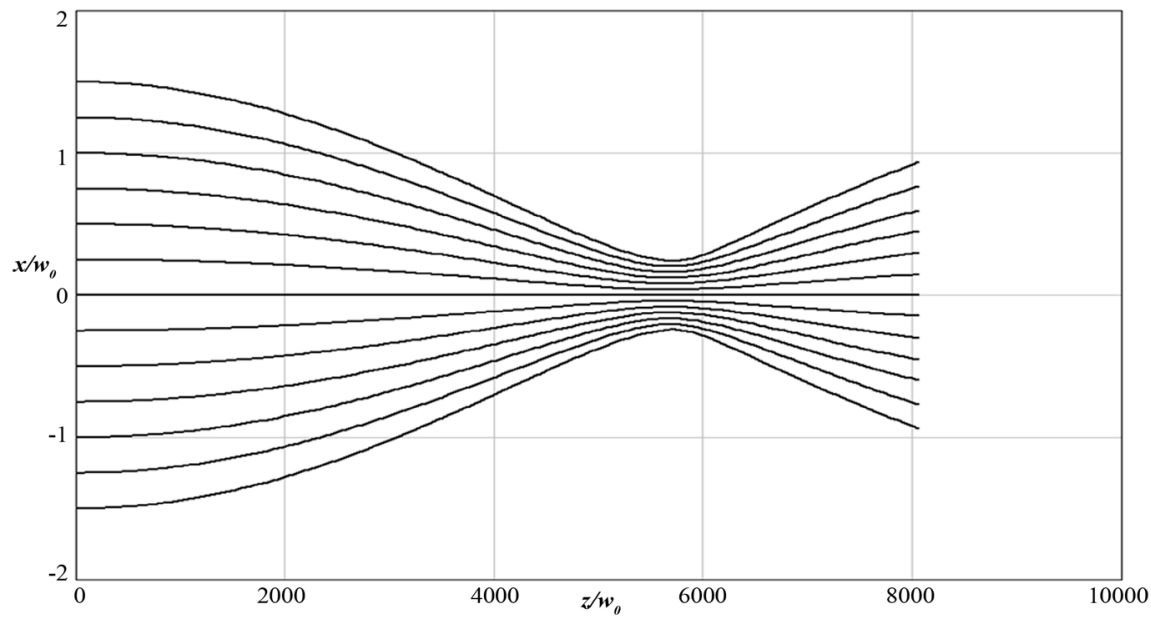

Figure 2. Lens-like potential: finite-focusing due to Wave Potential. 
Mechanics, by dropping from the system AII-1 the Wave Potential term $W(\boldsymbol{r}, E)$, is seen to be replaced by a finite focal waist (Figure 2) in Wave Mechanics, when the diffractive role of $W(\boldsymbol{r}, E)$ is taken into account.

\section{Time-Dependent Equations}

We could stop here, since the wave-particle duality is already adequately described, as we have shown, by the energy-dependent (and time-independent) Helmholtz-like Equation (18) and Equation (20) and by their dynamic trajectory systems AII-1 and AII-2.

Because, however, of the history itself of Quantum Mechanics, it's interesting to remind that two time-dependent equations [18] [19] may be obtained, making use of Equation (13), from Equation (18), in the form, respectively, of the usual-looking wave equation

$$
\nabla^{2} \psi=\frac{2 m}{E^{2}}(E-V) \frac{\partial^{2} \psi}{\partial t^{2}}
$$

with a phase velocity $E / \sqrt{2 m(E-V)}$, and of the unusual-looking, and energy independent, equation

$$
i \hbar \frac{\partial \psi}{\partial t}=-\frac{\hbar^{2}}{2 m} \nabla^{2} \psi+V(\boldsymbol{r}) \psi .
$$

which is the so-called "time-dependent" Schrödinger equation. Equation (26) was adopted, as is well known [18] [19], as the most significant generalization of Equation (18).

Referring - in order to fix ideas-to a discrete energy spectrum of Equation (18), and defining both the eigen-frequencies $\omega_{n} \equiv E_{n} / \hbar$ and the eigen-functions

$$
\psi_{n}(\boldsymbol{r}, t)=u_{n}(\boldsymbol{r}) \mathrm{e}^{-i E_{n} t / \hbar},
$$

any linear superposition (with arbitrary constant coefficients $c_{n}$ ) of the form

$$
\psi(\boldsymbol{r}, t)=\sum_{n} c_{n} \psi_{n}(\boldsymbol{r}, t),
$$

turns out to be a solution of Equation (26), splitting it into a time-evolving superposition of Helmholtz equations, in the form

$$
\sum_{n} c_{n} \mathrm{e}^{-i \frac{E_{n}}{\hbar} t}\left\{\nabla^{2} u_{n}(\boldsymbol{r})+\frac{2 m}{\hbar^{2}}\left[E_{n}-V(\boldsymbol{r})\right] u_{n}(\boldsymbol{r})\right\}=0 .
$$

The function (28) is a weighted average performed over the whole set of eigen-functions $\psi_{n}(\boldsymbol{r}, t)$, representing a particular "packet" of wave-trains. As we know from Section 3, however, each eigen-function $u_{n}(\boldsymbol{r})$ has its own trajectories, leading in general to the progressive space-dispersion of any wave-packet. The group velocity of a wave-packet takes on, indeed, the suggestive form

$$
\boldsymbol{v}_{g} \equiv \frac{\mathrm{d} \omega}{\mathrm{d} \boldsymbol{k}}=\frac{\mathrm{d}(E / \hbar)}{\mathrm{d}(\boldsymbol{p} / \hbar)}=\frac{\mathrm{d}\left(p^{2} / 2 m\right)}{\mathrm{d} \boldsymbol{p}}=\frac{\boldsymbol{p}}{m},
$$

referring to the packet center and apparently coinciding with the particle velocity, but obtained for a progressively diverging range around $p$. In Born's words 
[23], indeed, "it's very attractive to interpret a particle of matter as a wave-packet due to the superposition of a number of wave trains. But this tentative interpretation comes up against insurmountable difficulties, since a wave-packet of this kind is in general very soon dissipated". Let us recall, by comparison, that in our classical Hamiltonian case AII-1, holding for point-like particles, we have from Equation (21) an exact particle velocity

$$
\frac{\mathrm{d} \boldsymbol{r}}{\mathrm{d} t}=\frac{\partial H}{\partial \boldsymbol{p}} \equiv \frac{\boldsymbol{p}}{m}
$$

without any dispersion.

Born's proposal [24], giving rise to SQM, was to view the function (28) (which was called "Born Wave-Function") as representing a unique physical quantity carrying (before observation) the most complete information about the possible state of a particle, ranging in its full set of eigenstates, according to the (duly normalized) probabilities $\left|c_{n}\right|^{2}$. The continuous evolution of the Born Wave-Function was assumed, moreover, to (discontinuously) collapse into the energy eigen-value observed by the experimental set-up.

Born's Primary Assumption, leaving no room to physical intuition and discussion, was therefore given - as we said in the Introduction - by the "time-dependent" Schrödinger Equation (26) itself, to be viewed in a probabilistic perspective.

In the Bohmian approach [25] [26], whose state of art is thoroughly described in [27]-[32], Born's Wave-Function was written in the form

$$
\psi(\boldsymbol{r}, t) \equiv \sum_{n} c_{n} \psi_{n}(\boldsymbol{r}, t)=R(\boldsymbol{r}, t) \mathrm{e}^{i G(\boldsymbol{r}, t) / \hbar}
$$

with real $R(\boldsymbol{r}, t)$ and $G(\boldsymbol{r}, t)$, and $R^{2} \equiv|\psi|^{2} \equiv \psi \psi^{*}$. The expression (32) was "shaped" on de Broglie's Equation (16), with the aim of viewing Born's "Wave-Function" as a generalized de Broglie's wave, participating, possibly, in Davisson-Germer's detectability. The role of de Broglie's Primary Assumption $\boldsymbol{p}=\hbar \boldsymbol{k}$ (and of de Broglie himself as founding father of Wave Mechanics) was therefore overshadowed, and somewhat diminished, by this cooptation. Concerning the function (32), it's easily verified that

$$
\frac{\nabla G(\boldsymbol{r}, t)}{m} \equiv \frac{\hbar}{2 m i} \frac{\psi^{*} \nabla \psi-\psi \nabla \psi^{*}}{\psi \psi^{*}} .
$$

Since, in SQM [18] [19], the fluid-like probability current density is given by the expression $\boldsymbol{J} \equiv \frac{\hbar}{2 m i}\left(\psi^{*} \nabla \psi-\psi \nabla \psi^{*}\right)$, the term $\frac{\nabla G(\boldsymbol{r}, t)}{m}$ is seen to coincide with the velocity $\boldsymbol{v}_{\text {prob }}(\boldsymbol{r}, t)$ at which the fluid-like probability density is transported. That is why, in the Bohmian theory, the particle Guidance Law is assumed in the form

$$
\frac{\mathrm{d} \boldsymbol{r}}{\mathrm{d} t}=\boldsymbol{v}_{\text {prob }}(\boldsymbol{r}, t) \equiv \frac{\nabla G(\boldsymbol{r}, t)}{m},
$$

where the particle is represented by a wave-packet centered at $\boldsymbol{r}$.

This choice gives rise to a hydrodynamic visualisation of SQM, utterly different both from the dynamic "Guidance Law" that de Broglie was looking for and 
from our own Guidance Law (21).

\section{Discussion and Conclusions}

As we wrote in the Introduction, the direct assumption of Schrödinger's equations (together with their probabilistic interpretation) as axiomatic Primary Assumptions of Quantum Mechanics doesn't help the intuitive understanding of its standard interpretation and of its possible alternatives. Starting, on the contrary, from de Broglie's Assumption $\boldsymbol{p}=\hbar \boldsymbol{k}$ (in its complete vectorial form) is quite helpful both for the physical intuition of Wave Mechanics and for its subsequent development.

Both in his juvenile years [10] [11] and in his later papers [1] [12] [20] [21] [22] de Broglie had clear in mind the problem of localizing and addressing the particles along a classical-looking path, starting from assigned launching conditions and according to a consistent Guidance Law. No forward step could be performed, however, before the discovery [3] of the Hamiltonian ray-tracing properties of Helmholtz-like equations.

As we have shown, the desired Guidance Law is given by Equation (21), duly accompanied by the full Hamiltonian systems AII-1 and/or AII-2. Their time-integration tells us that an exact and classical-looking point-particle dynamics, guided by matter waves, is both possible and easily practicable, contrary to the assertion of an intrinsically probabilistic and indeterministic nature of physical reality: an assertion whose extrapolations lead to a host of quantum paradoxes [33] [34], including doubts about the physical reality itself of material particles.

As far as the natural development of the present study is concerned, we are presently working on its extension to many-particle applications. Here, too, de Broglie's juvenile work appears to provide an essential contribution, in striking contrast with the SQM route. At the Solvay Conference of 1927 the father of Wave Mechanics happened to write, in fact [35]: "It appears to us certain that if one wants to physically represent the evolution of a system of $N$ corpuscles, one must consider the propagation of $N$ waves in space, each of the $N$ propagations being determined by the action of the $N-1$ corpuscles connected to the other waves. (...) Contrary to what happens for a single material point, it does not appear easy to find a single wave that would define the motion of a system taking Relativity into account".

\section{Conflicts of Interest}

The authors declare that there is no conflict of interest concerning the present paper.

\section{References}

[1] de Broglie, L. (1959) L'interprétation de la Mécanique Ondulatoire. Le Journal de Physique et le Radium, 20, 963-979.

https://doi.org/10.1051/jphysrad:019590020012096300 
[2] Pauling, L. and Wilson Jr., E.B. (1935) Introduction to Quantum Mechanics. McGraw-Hill Company, Inc., NewYork, London.

[3] Orefice, A., Giovanelli, R. and Ditto, D. (2009) Complete Hamiltonian Description of Wave-Like Features in Classical and Quantum Physics. Foundations of Physics, 39, 256. https://doi.org/10.1007/s10701-009-9280-2

[4] Orefice, A., Giovanelli, R. and Ditto, D. (2012) Beyond the Eikonal Approximation in Classical Optics and Quantum Physics. In: Oriols, X. and Mompart, J., Eds., Applied Bohmian Mechanics: From Nanoscale Systems to Cosmology, Chapter: 7, PAN Stanford Publishing, Singapore, 425-453

[5] Orefice, A., Giovanelli, R. and Ditto, D. (2013) A Non-Probabilistic Insight into Wave Mechanic. Annales de la Fondation Louis de Broglie, 38, 7-31.

[6] Orefice, A., Giovanelli, R. and Ditto, D. (2015) From Classical to Wave-Mechanical Dynamics. Annales de la Fondation Louis de Broglie, 40, 95.

[7] Orefice, A., Giovanelli, R. and Ditto, D. (2015) Is Wave Mechanics Consistent with Classical Logic? Physics Essays, 28, 515-521.

https://doi.org/10.4006/0836-1398-28.4.515

[8] Orefice, A., Giovanelli, R. and Ditto, D. (2018) Dynamics of Wave-Particle Duality. Journal of Applied Mathematics and Physics, 6, 1840.

[9] Goldstein, H. (1965) Classical Mechanics. Addison-Welsey, Boston.

[10] de Broglie, L. (1924) A Tentative Theory of Light Quanta. The London, Edinburgh, and Dublin Philosophical Magazine and Journal of Science, 47, 446-458. https://doi.org/10.1080/14786442408634378

[11] de Broglie, L. (1925) Recherches sur la théorie des quanta. Annales de Physique, 10, 22.

[12] de Broglie, L. (1965) La nature ondulatoire del'électron. Nobel Lectures in Physics 1922-1941, Elsevier Publ. Co., Amsterdam, 244.

[13] Davisson, C.J. and Germer, L.H. (1927) The Scattering of Electrons by a Single Crystal of Nickel. Nature, 119, 558-560. https://doi.org/10.1038/119558a0

[14] Schrödinger, E. (1926) Quantisierung als Eigenwertproblem I. Annalen der Physik, 79, 361. https://doi.org/10.1002/andp.19263840404

[15] Schrödinger, E. (1926) Quantisierung als Eigenwertproblem II. Annalen der Physik, 79, 489. https://doi.org/10.1002/andp.19263840602

[16] Schrödinger, E. (1926) Quantisierung als Eigenwertproblem III. Annalen der Physik, 80, 437. https://doi.org/10.1002/andp.19263851302

[17] Schrödinger, E. (1926) Quantisierung als Eigenwertproblem IV. Annalen der Physik, 81, 109. https://doi.org/10.1002/andp.19263861802

[18] Persico, E. (1950) Fundamentals of Quantum Mechanics. Prentice-Hall, Inc., Upper Saddle River.

[19] Messiah, A. (1959) Mécanique Quantique, Dunod.

[20] de Broglie, L. (1956) Une tentative d'interprétation causale et non-linéaire de la Mécanique Ondulatoire: La théorie de la double solution. Gauthier-Villars, Paris.

[21] de Broglie, L. (1971) L'interprétation de la Mécanique ondulatoire par la théorie de la double solution. In: d'Espagnat, B., Ed., Foundations of Quantum Mechanics, Academic Press, New York, 345-367.

[22] de Broglie, L. (1987) Interpretation of Quantum Mechanics by the Double Solution Theory. Annales de la Fondation Louis de Broglie, 12, 1.

[23] Born, M. (1935) Atomic Physics. Blackie \& Son Ltd., London. 
[24] Born, M. (1926) Quantenmechanik der Stoßvorgänge. Zeitschrift für Physik, 38, 803-827. https://doi.org/10.1007/BF01397184

[25] Bohm, D.J. (1952) A Suggested Interpretation of the Quantum Theory in Terms of “Hidden” Variables I. Physical Review, 85, 166. https://doi.org/10.1103/PhysRev.85.166

[26] Bohm, D.J. (1952) A Suggested Interpretation of the Quantum Theory in Terms of “Hidden" Variables II. Physical Review, 85, 180. https://doi.org/10.1103/PhysRev.85.180

[27] Holland, P.R. (1992) The Quantum Theory of Motion. Cambridge University Press, Cambridge.

[28] Wyatt, R.E. (2005) Quantum Dynamics with Trajectories: Introduction to Quantum Hydrodynamics. Springer, Berlin.

[29] Dürr, D. and Teufel, S. (2009) Bohmian Mechanics. Springer-Verlag, Berlin.

[30] Oriols, X. and Mompart, J. (2012) Applied Bohmian Mechanics: From Nanoscale Systems to Cosmology. Pan Stanford Publishing.

[31] Sanz, A.S. and Miret-Artès, S. (2012-2014) A Trajectory Description of Quantum Processes. Vol. I-II, Springer, Berlin.

[32] Benseny, A., Albareda, G., Sanz, A.S., Mompart, J. and Oriols, X. (2014) Applied Bohmian Mechanics. The European Physical Journal D, 68, 286. https://doi.org/10.1140/epjd/e2014-50222-4

[33] Aharonov, Y. and Rohrlich, D. (2005) Quantum Paradoxes: Quantum Theory for the Perplexed. Wiley, Hoboken. https://doi.org/10.1002/9783527619115

[34] Hardy, L. (1992) Quantum Mechanics, Local Realistic Theories and Lorentz Invariant Theories. Physical Review Letters, 68, 2981. https://doi.org/10.1103/PhysRevLett.68.2981

[35] de Broglie, L. (1927) The New Dynamics of Quanta. In: Bacciagaluppi, G. and Valentini, A., Eds., The Book Quantum Theory at the Crossroads. Reconsidering the 1927 Solvay Conference, Part III, Cambridge University Press, Cambridge, 374. 


\section{Appendix I}

We report here the beginning of de Broglie's original text of the paper "L'interprétation de la Mécanique Ondulatoire" [1] written, in French, in 1959.

Dans mes premiers travaux sur la Mécanique ondulatoire, qui remontent à 1923, j'avais clairement aperçu qu'il fallait d'une façon générale associer au mouvement de tout corpuscule la propagation d'une onde. Mais l'onde homogène que j'avais été amené à considérer, et qui est devenue l'onde $\psi$ de la Mécanique ondulatoire usuelle, ne me paraissait pas décrire la réalité physique. (...) Ne donnant aucune prérogative particulière à aucun point de Pespace, elle n'était pas susceptible de représenter la position du corpuscule: tout au plus pouvait-on supposer, comme on le fit bientôt, qu'elle donnait par son carré la "probabilitè de présence" du corpuscule en chaque point (...).

Aucun physicien n'ignore aujourd'hui (1959) que la Mécanique ondulatoire a reçu depuis plus de trente ans une interprétation "purement probabiliste" dans laquelle I'onde associée au corpuscule n'est plus qu'une représentation de probabilité dependant de l'état de nos informations à son sujet, et susceptible de varier brusquement avec elles (réduction du paquet de probabilité au sens de Heisenberg), tandis que le corpuscule est conçu comme n'ayant pas de localization permanente dans lespace et, par suite, comme ne décrivant pas une trajectoire bien définie. Cette manière de concevoir le dualisme onde-corpuscule a reçu le nom de "complémentarité", notion assez peu précise que l'on a cherché à extrapoler, d'une façon un peu périlleuse, en dehors du domaine propre de la Physique.

Cette interprétation de la Mécanique ondulatoire, bien différente, je le rappellerai, de celle que j'avais envisagée au début de mes recherches, est due principalement à MM. Born, Bohr et Heisenberg dont les brillants travaux sont d'ailleurs dignes de la plus grande admiration. Elle a été assez rapidement adoptée par presque tous les théoriciens malgré les réserves expresses que faisaient à son sujet des esprits aussi éminents que MM. Einstein et Schrödinger et les objections qu'ils lui opposaient. Personnellement, après avoir proposé une interprétation tout à fait différente je me suis rallié à celle qui devenait “orthodoxe", et je l'ai enseignée pendant de longues années. Mais depuis 1951, à la suite notamment de tentatives faites à cette époque par MM. Bohm et Vigier, je me suis à nouveau demandé si ma première orientation vis-à-vis du problème posé par l'existence du dualisme onde-corpuscule n'était pas la bonne. Quelques années ont passé et il me semble que le moment est venu de faire une nouvelle mise au point de l'état de la question en tenant compte des progrès accomplis depuis mes exposés de 1953-1954.

Les objections les plus fortes que l'on peut élever contre l'interprétation actuellement admise de la Mécanique ondulatoire sont relatives à la non-localization du corpuscule dans cette interprétation. Elle admet, en effet, que, si l'état de nos connaissances sur un corpuscule est représenté par un train d'onde $\psi$ étendu, le corpuscule est présent dans tous les points de ce train d'ondes avec une 
probabilité égale à $|\psi|^{2}$ : cette présence pourrait être qualifiée de "potentielle" et c'est seulement au moment où nous constatons la présence du corpuscule en un point du train d'ondes par une observation que cette potentialité s'actualise, pour employer un langage de philosophes. Une telle conception se heurte à des difficultés qui ont été signalées avec force et de diverses manières par MM. Einstein et Schrödinger.

\section{Appendix II}

We report here (from [8]) the Hamiltonian systems of particle trajectories holding, respectively, in the non-relativistic and in the relativistic case.

\begin{tabular}{cc}
\multicolumn{1}{c}{$\begin{array}{c}\text { A II-1 } \\
\text { Non-Relativistic } \\
\text { Hamiltonian Trajectories }\end{array}$} & $\begin{array}{c}\text { AII-2 } \\
\text { Relativistic } \\
\text { Hamiltonian Trajectories }\end{array}$ \\
\hline$\left\{\begin{array}{l}\frac{\mathrm{d} \boldsymbol{r}}{\mathrm{d} t}=\frac{\partial H}{\partial \boldsymbol{p}} \equiv \frac{\boldsymbol{p}}{m} \\
\frac{\mathrm{d} \boldsymbol{p}}{\mathrm{d} t}=-\frac{\partial H}{\partial \boldsymbol{r}} \equiv-\nabla[V(\boldsymbol{r})+W(\boldsymbol{r}, E)] \\
\nabla \cdot\left(R^{2} \boldsymbol{p}\right)=0 \\
|\boldsymbol{p}(t=0)| \equiv p_{0}=\sqrt{2 m E}\end{array}\right.$ & $\begin{array}{l}\frac{\mathrm{d} \boldsymbol{r}}{\mathrm{d} t}=\frac{\partial H}{\partial \boldsymbol{p}} \equiv \frac{c^{2} \boldsymbol{p}}{E-V(\boldsymbol{r})} \\
\frac{\mathrm{d} \boldsymbol{p}}{\mathrm{d} t}=-\frac{\partial H}{\partial \boldsymbol{r}} \equiv-\nabla V(\boldsymbol{r})-\frac{E}{E-V(\boldsymbol{r})} \nabla W(\boldsymbol{r}, E) \\
\nabla \cdot\left(R^{2} \boldsymbol{p}\right)=0 \\
|\boldsymbol{p}(t=0)| \equiv p_{0}=\sqrt{(E / c)^{2}-\left(m_{0} c\right)^{2}} \\
W(\boldsymbol{r}, E)=-\frac{\hbar^{2}}{2 m} \frac{\nabla^{2} R(\boldsymbol{r}, E)}{R(\boldsymbol{r}, E)}\end{array}$ \\
\end{tabular}

